\title{
X-ray diffraction as a tool for the determination of the structure of double-walled carbon nanotube batches
}

\author{
J. Cambedouzou, ${ }^{1, *}$ M. Chorro, ${ }^{1}$ R. Almairac, ${ }^{2}$ L. Noé,${ }^{3}$ E. Flahaut, ${ }^{4}$ S. Rols,${ }^{5}$ M. Monthioux, ${ }^{3}$ and P. Launois ${ }^{1}$ \\ ${ }^{1}$ Laboratoire de Physique des Solides, Université Paris Sud XI, UMR CNRS 8502, F-91405 Orsay, France \\ ${ }^{2}$ Laboratoire des Collö̈des, Verres et Nanomatériaux, Université Montpellier II, UMR CNRS 5587, F-34095 Montpellier, France \\ ${ }^{3}$ Centre d'Elaboration de Matériaux et d'Etudes Structurales, Université Toulouse III, UPR CNRS 8011, F-31055 Toulouse, France \\ ${ }^{4}$ CNRS; Institut Carnot Cirimat; F-31062 Toulouse, France \\ ${ }^{5}$ Institut Laue Langevin, F-38042 Grenoble, France
}

(Received 16 January 2009; revised manuscript received 27 April 2009; published 20 May 2009)

\begin{abstract}
The average structure of double-walled carbon nanotube (DWCNT) samples can be determined by x-ray diffraction (XRD). We present a formalism that allows XRD patterns of DWCNTs to be simulated and we give researchers the tools needed to perform these calculations themselves. Simulations of XRD patterns within this formalism are compared to experimental data obtained on two different DWCNT samples, produced by chemical vapor deposition or by peapod conversion (i.e., high-temperature peapod annealing). For each sample, we are able to determine structural aspects such as the number of walls, the diameter distribution of inner and outer tubes, the intertube spacing, and the bundled structure.
\end{abstract}

DOI: 10.1103/PhysRevB.79.195423

PACS number(s): $61.46 . \mathrm{Np}$

\section{INTRODUCTION}

Among the numerous carbon nanotube-based materials synthesized for the last 10 years, double-walled carbon nanotubes (DWCNTs) are certainly one of the most attractive, particularly due to their potential applications in the field of nanoelectronics. Cylindrical nanocapacitors ${ }^{1,2}$ and coaxial nanocables ${ }^{3}$ - possibly electronically tunable ${ }^{4}$-are two of the most promoted uses of DWCNTs. In addition to these potential applications, DWCNTs are of high fundamental interest as they stand at the frontier between single-walled carbon nanotubes (SWCNTs) and multiwalled carbon nanotubes (MWCNTs) and they therefore can be considered as a model system to study wall to wall interactions ${ }^{5}$ and frustration effects due to incommensurability between the atomic structure of external and internal walls. ${ }^{6}$

In order to obtain DWCNTs, two major synthesis routes are known, namely, the "direct synthesis" route, using regular carbon nanotubes synthesis methods such as catalytic chemical vapor deposition (CCVD) (Refs. 7 and 8) or electric arc plasmas" and the "peapod conversion" route. ${ }^{3,10}$ The first route allows the direct catalyst-assisted synthesis of DWCNTs from the decomposition of carbonaceous gas precursors (CCVD) or the sublimation of graphite anodes (arc discharge). Specifically, the CCVD method is suited to largescale synthesis ${ }^{7}$ and is therefore very promising for the development of applications involving DWCNTs. The second route is less direct since it involves a first stage of fullerene intercalation inside single-walled carbon nanotubes ${ }^{11,12}$ to an intercalation rate as high as possible and then heating the so-called peapods to a temperature of $1000-1300{ }^{\circ} \mathrm{C}$. Given the important difference between the two ways of synthesizing DWCNTs, it would not be surprising to observe differences between the structures of DWCNTs coming from different direct routes or from the peapod conversion route.

Transmission electron microscopy (TEM) observations have demonstrated that, depending on the synthesis conditions, CCVD-produced DWCNTs can remain mainly isolated or occasionally form small bundles where tubes are heterogeneous. ${ }^{7}$ In some exceptional cases, DWCNTs assembled in bundles can even have the same helicities, as was shown using electron-diffraction technique. ${ }^{13}$ In this paper, we will focus on samples made as described in Ref. 7 when we refer to CCVD-produced samples. The analysis of about 100 nanotubes observed by TEM shows that tubes are isolated, with a diameter distribution, centered around $20 \AA$ for the external tube, which is very large [full width at half maximum $(F W H M)=12 \AA]$. At the opposite, peapods conversion leads to the formation of DWCNTs with a narrow diameter distribution $(\mathrm{FWHM}=2 \AA$ ) centered around $14 \AA$ for the external tube and these DWCNTs are assembled in large bundles. ${ }^{3}$ Pairs of tubes in DWCNTs can also be evidenced through Raman radial breathing mode (RBM) frequencies. ${ }^{1,2,8,14}$ However, this technique is not straightforward for giving a quantitative characterization of a sample due to resonance effects, which make a large number of nanotubes invisible in the Raman response of a sample. Moreover, the penetration depth of visible lasers into nanotubes is too low to allow a global characterization of a macroscopic sample.

In order to get a statistically relevant image of a DWCNT sample, x-ray diffraction (XRD) appears as an interesting method since it can probe milligram-scale samples, without any specific selection between carbon nanotubes in the sample. This technique has already been used in order to follow the peapods conversion into DWCNTs, ${ }^{15,16}$ but the calculations of diffraction patterns were not fully rigorous as ad hoc Gaussian and Lorentzian functions were used to simulate Bragg peaks. Such an approximation, which can only be used when diffraction peaks are rather well defined, that is for large bundles of nanotubes with a narrow diameter distribution does not apply to other physical cases corresponding to unbundled DWCNTs (or bundles of small size) and/or to wide diameter distribution within the sample. We intend giving here detailed indications on how diffraction should be treated to obtain an accurate description of 
DWCNT samples, from CCVD samples consisting of DWCNT with many different diameters to those resulting from peapods conversion, which have already been studied using X-ray scattering ${ }^{15,16}$ and correspond to the "simplest" case with relatively well-defined diffraction peaks. We intend to give experimentalists readily usable tools to perform fast characterizations of their DWCNT samples, whatever their structural characteristics. In the first part, we will describe how simulations of diffraction patterns can be performed in order to fully understand experimental diffraction profiles of DWCNT samples from CCVD and peapods conversion. In the second part, we will illustrate the application of the calculations developed in the first part on samples of DWCNTs synthesized by both routes.

\section{RESULTS AND DISCUSSION}

\section{A. Calculations}

\section{Isolated nanotubes}

The formalism we use in our calculations is derived from that initially developed by Thess et al., ${ }^{17}$ involving homogeneous cylinders with a constant surface density of scatterers, and then further improved to better adapt to the case of single-walled carbon nanotubes ${ }^{18}$ and peapods. ${ }^{19,20}$ With the information on the relative position of carbon atoms constitutive of nanotubes' walls being lost, the hypothesis of homogeneous scattering surfaces limits the relevance of our calculations to scattering vectors $\mathbf{Q}$ with modulus lower than $2.5 \AA^{-1}$. The diffraction profile of a powdered sample is given by the square of the Fourier transform of the spatial configuration of scattering surfaces of the nanotubes, for all relative orientations between the nanotubes and the scattering vector. Calculations are performed using a homemade program.

Let us first consider a powder of identical individual DWCNTs, having an inner diameter $D_{i}$ and an outer diameter $D_{o}$. It can easily be shown, following, e.g., the formalism developed in Appendix A in Ref. 19 or in Ref. 21, that the scattering intensity $I_{\mathrm{DW}}$ of a single DWCNT may be written as

$$
I_{\mathrm{DW}}(\vec{Q}) \propto\left\{\sigma f_{c}(Q)\left[D_{i} J_{0}\left(\frac{Q D_{i}}{2}\right)+D_{o} J_{0}\left(\frac{Q D_{o}}{2}\right)\right]\right\}^{2} \delta\left(Q_{z}\right),
$$

where $\sigma$ represents the surface density of scatterers on a nanotube wall $\left(\sigma \sim 0.38\right.$ atom $\left./ \AA^{2}\right), f_{c}$ is the carbon form factor for $\mathrm{x}$ rays, $J_{0}$ is the cylindrical Bessel function of order zero, $\delta$ is the Dirac distribution, and $Q_{z}$ is the component of the wave vector along the tube axis. The intensity of a powder of such an object is given by

$$
I(Q) \propto \frac{\iint I_{\mathrm{DW}}(\vec{Q}) d^{2} S_{Q}}{4 \pi Q^{2}},
$$

where the integration is performed over the sphere of radius $Q$ and $d^{2} S_{Q}$ is a surface element of this sphere. It leads to the following expression:

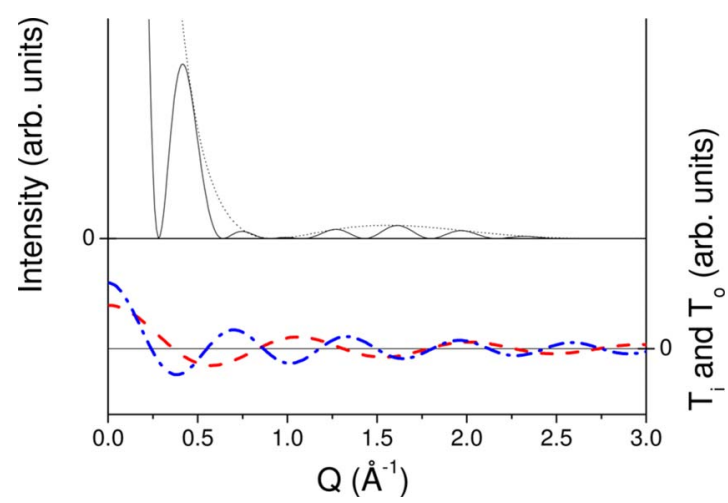

FIG. 1. (Color online) Plain line: calculated intensity of a powder of isolated DWCNTs with $D_{i}=13.2 \AA$ and $D_{o}=20 \AA\left(D_{m}\right.$ $=16.6 \AA$ and $d R=3.4 \AA$ ). Dotted line: envelope function. Dashed line: calculation of the $T_{i}$ term involved in the intensity scattered by the inner tube. Dashed-dotted line: calculation of the $T_{o}$ term involved in the intensity scattered by the outer tube.

$$
I(Q) \propto \frac{\sigma^{2} f_{c}^{2}}{2 Q}\left[D_{i} J_{0}\left(\frac{Q D_{i}}{2}\right)+D_{o} J_{0}\left(\frac{Q D_{o}}{2}\right)\right]^{2} .
$$

The result of this calculation can be seen in Fig. 1 for a DWCNT with $D_{i}=13.2 \AA$ and $D_{o}=D_{i}+2 d R=13.2+2 \times 3.4$ $=20 \AA$, with $d R$ being the van der Waals distance between the inner and the outer tubes. The mean diameter of the tube, defined as $D_{m}=\left(D_{i}+D_{o}\right) / 2$, is equal to $16.6 \AA$. The scattered intensity consists of oscillations within an envelope function (see below), with the latter being minimum around 0.9 and $2.8 \AA^{-1}$.

The origin of the modulation of the oscillations can be deduced from the observation of the terms $T_{i}=D_{i} J_{0}\left(\frac{Q D_{i}}{2}\right)$ and $T_{o}=D_{o} J_{0}\left(\frac{Q D_{o}}{2}\right)$, respectively, related to the inner and the outer tube and involved in the calculation of the scattered intensity. One observes in Fig. 1 that the period of the Bessel function corresponding to the tube of the smallest diameter is larger than that of the largest nanotube. In the area where the oscillations are out of phase (e.g., between 0.7 and $1.1 \AA^{-1}$ ), the intensity resulting from the squared modulus of $\left(T_{i}+T_{o}\right)$ is minimum and it is maximum where the oscillations are in phase (e.g., between 1.6 and $2.3 \AA^{-1}$ ).

Figure 2 illustrates the effects of changing the inner- and outer-tube diameters keeping the intertube distance $d R$ equal to $3.4 \AA$. When the mean diameter of the DWCNT is increased, the oscillation period decreases in good agreement with the behavior of the zero-order Bessel function associated with each wall (see Fig. 1). However, it is interesting to remark that the shape of the envelope function appears unchanged: the positions of the minima and maxima of the envelope function depend only on the distance $d R$ between the walls. It has indeed been shown in the former paragraph that it was directed by interferences between the Bessel functions associated with each nanotube wall.

Figure 3 shows how the diffracted intensity evolves when $d R$ is varied between 3 and $3.8 \AA$, keeping the mean diameter constant. The positions of the zeros of the oscillations are unchanged: they thus depend on the value of the mean 


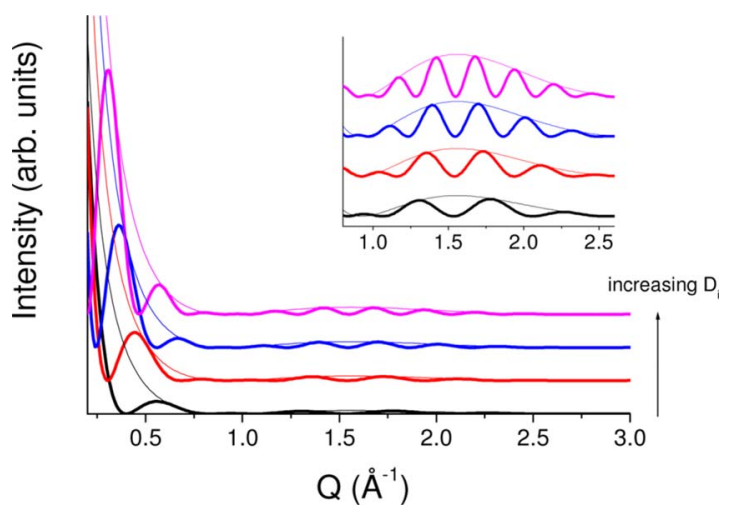

FIG. 2. (Color online) Calculated intensity of a powder of isolated DWCNTs with an interwall distance $d R$ of $3.4 \AA$ and innertube diameters of (from bottom to top) 8, 12, 16, and $20 \AA$. Curves are translated vertically for clarity and their envelope functions are drawn. Inset: magnification of the $\left[0.8-2.6 \AA^{-1}\right]$ range.

diameter of the tube. At the opposite, the positions of the zeros and of the second maximum of the envelope curve are clearly shifted toward the lower $Q$ values when the interwall distance $d R$ is increased.

The analytical description of this envelope curve can be achieved by considering the asymptotical development of the $\mathrm{J}_{0}$ Bessel function, ${ }^{22}$

$$
J_{0}(x) \approx \sqrt{\frac{2}{\pi x}} \cos \left(x-\frac{\pi}{4}\right) .
$$

This approximation is valid for $x>0.9$, i.e., $Q R>0.9$. These conditions are respected while tubes with a radius larger than $3 \AA$ are considered for $Q$ values greater than $0.3 \AA^{-1}$. Within this approximation, the intensity of a powder of isolated DWCNTs may be written from Eq. (3) as

$$
I(Q) \propto \frac{2 \sigma^{2} f_{c}^{2}}{\pi Q^{2}}\left[\sqrt{D_{i}} \cos \left(\frac{Q D_{i}}{2}-\frac{\pi}{4}\right)+\sqrt{D_{o}} \cos \left(\frac{Q D_{o}}{2}-\frac{\pi}{4}\right)\right]^{2} .
$$

One derives from the above expression the following one, using classical trigonometric relations and limited develop-

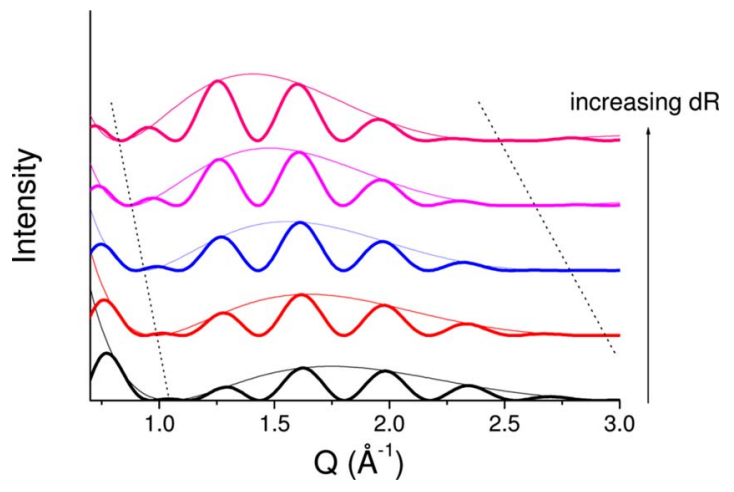

FIG. 3. (Color online) Calculated intensity of a powder of isolated DWCNTs with a mean diameter of $16.6 \AA$ and with interwall distances $d R$ of (from bottom to top) 3, 3.2, 3.4, 3.6, and $3.8 \AA$. Curves are translated vertically for clarity and their envelope functions are drawn. Dotted lines are guide to the eyes.

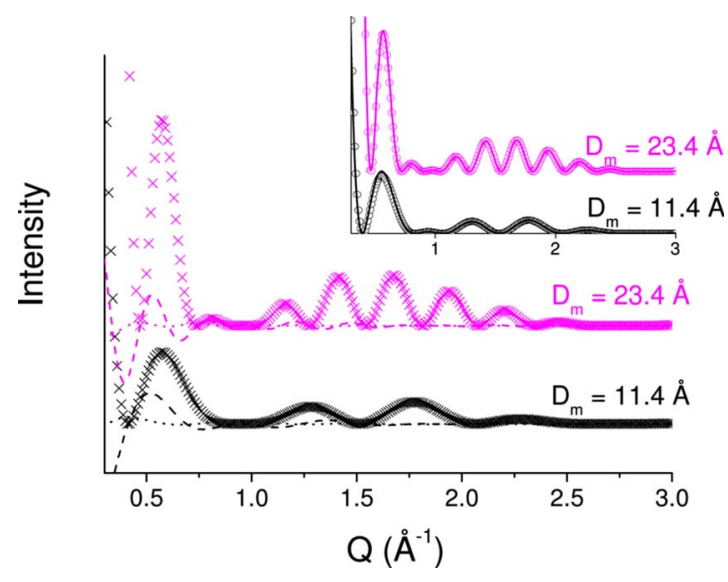

FIG. 4. (Color online) Calculated intensity of a powder of isolated DWCNTs with an interwall distance $d R$ of $3.4 \AA$ and innertube diameters of 8 and $20 \AA$, for the bottom and upper curves, respectively. Curves are translated vertically for clarity. Crosses: first term of Eq. (6), dashed line: second term, and dotted line: third term. Inset: calculated intensities of the same powders using Eq. (3) (open circles) and Eq. (6) (solid line).

ments to the order 2 of the function $\sqrt{1-\left(\frac{d R}{D_{m}}\right)^{2}}$ that appears in the analytical development,

$$
\begin{aligned}
I(Q) \propto & \frac{2 \sigma^{2} f_{c}^{2} D_{m}}{\pi Q^{2}}\left\{4 \cos ^{2}\left(\frac{Q D_{m}}{2}-\frac{\pi}{4}\right) \cos ^{2}\left(Q \frac{d R}{2}\right)\right. \\
& -\frac{d R}{D_{m}} \sin \left(Q D_{m}-\frac{\pi}{2}\right) \sin (Q d R) \\
& \left.-\frac{d R^{2}}{2 D_{m}^{2}}\left[\cos \left(Q D_{m}-\frac{\pi}{2}\right)+\cos (Q d R)\right]\right\} .
\end{aligned}
$$

This expression contains three terms that are separately plotted in Fig. 4 in the case of two powders of DWCNTs with $D_{m}=11.4$ and $23.4 \AA\left(D_{i}=8\right.$ and $\left.20 \AA\right)$, respectively, and $d R=3.4 \AA$ in both cases. It allows explaining analytically all the features of the intensity calculated for a powder of DWCNTs.

In Fig. 4 inset, a comparison between intensities calculated from Eqs. (3) and (6) shows that the limited development to the order 2 in $d R / D_{m}$ is a good approximation, even for relatively small diameters. It is also obvious in Fig. 4 that the most important term in Eq. (6) is the first one; the other terms are negligible above $1 \AA^{-1}$. This first term contains a periodic function of period $2 \pi / D_{m}$, which is actually the period of the short oscillations. On one hand, this period is independent on the interwall distance $d R$ but is related to the mean diameter of DWCNTs $D_{m}$, which is in good agreement with our previous results. On the other hand, the first term is modulated by an envelope function defined as $F_{\text {env }}(Q)$ $=8 \sigma^{2} \frac{f_{c}^{2} D_{m}}{\pi Q^{2}} \cos ^{2}\left(Q \frac{d R}{2}\right)$, which only depends on the intertube spacing and which reaches zero for $Q=\pi / d R$ then $Q$ $=3 \pi / d R$. For $d R=3.4 \AA$, these values are 0.92 and $2.78 \AA^{-1}$, respectively. They were indeed found above to be independent of the DWCNT mean diameter and to depend only on the $d R$ interwall spacing. 
It must be emphasized that the study of this envelope curve presents a strong interest in the perspective of fitting experimental diffraction patterns from real samples. Indeed, the period of the narrow oscillations from DWCNTs changes depending on the DWCNT diameter. If a sample presents a large diameter distribution, it will not be possible to distinguish these oscillations anymore since there will be a merging of the signal from each DWCNT. As a result, the only remaining feature in the diffraction pattern will be the envelope curve, which is the same for all nanotube diameters. Consequently, the envelope curves can roughly be considered as the diffraction signal of a sample presenting a wide tube diameter distribution.

In the following, we focus on how the diffraction pattern evolves when tubes containing $N=3,4$, or 5 walls are considered. Let us postulate that these MWCNTs are constituted by coaxial nanotubes with a fixed interwall spacing $d R$. The diameter of the $n$th tube writes $D_{n}=D_{i}+2(n-1) d R$, where $n$ goes from 1 to $N$, and the diffracted intensity, in the limits of the asymptotic development of $J_{0}$ Bessel functions, is proportional to

$$
I(Q) \propto D_{m}\left[\cos \left(\frac{Q D_{i}}{2}-\frac{\pi}{4}\right)+\cdots+\cos \left(\frac{Q D_{N}}{2}-\frac{\pi}{4}\right)\right]^{2},
$$

where $D_{m}$ is still the mean diameter $\left[D_{m}=\left(D_{1}+D_{N}\right) / 2\right]$. The above calculations for DWCNTs allowed us to show that the most important term in Eq. (6) corresponds to the development of order zero in $d R / D_{m}$, so we have restricted the calculations to this term. The sum of cosine functions may also be written as $\cos \left(\frac{Q D_{m}}{2}-\frac{\pi}{4}\right)\left[\sin \left(\frac{N Q d R}{2}\right) / \sin \left(\frac{Q d R}{2}\right)\right]$ and therefore we can write the intensity of a powder of small MWCNTs renormalized to the number $N$ of walls (with $N=2,3,4$, or 5) as

$$
I(Q) \propto \frac{\sigma^{2} f_{c}^{2} D_{m}}{\pi Q^{2}} \cos ^{2}\left(\frac{Q D_{m}}{2}-\frac{\pi}{4}\right) \frac{1}{N}\left[\frac{\sin \left(\frac{N Q d R}{2}\right)}{\sin \left(\frac{Q d R}{2}\right)}\right]^{2} .
$$

We show in Fig. 5 the envelope curves of MWCNT given by the formula

$$
\left(f_{c}^{2} D_{m} / \pi Q^{2} N\right)[\sin (N Q d R / 2) / \sin (Q d R / 2)]^{2} .
$$

When the number of walls is increased, two remarkable effects must be pointed out in the calculated diffraction pattern of MWCNTs. (i) The position of the maximum of the most intense oscillation between 1 and $2 \AA^{-1}$ shifts from about $1.56 \AA^{-1}$ for $N=2$ (DWCNTs) to $1.85 \AA^{-1}$. This latter position corresponds to that of the Bragg reflection on the $\{001\}$ planes of turbostratic graphite or of classical MWCNTs $(Q$ $\left.=\frac{2 \pi}{d R}\right)$. (ii) The width of this maximum sharpens. We plotted the position of the maximum and the FWHM of this feature versus the number of walls in Fig. 6. At first sight, the FWHM of the diffraction feature located around $1.8 \AA^{-1}$ follows a $1 / N$ dependence, in agreement with what is expected from the Scherrer law. ${ }^{23}$ This result, observed after having fitted the curves calculated from Eq. (9), can also be derived

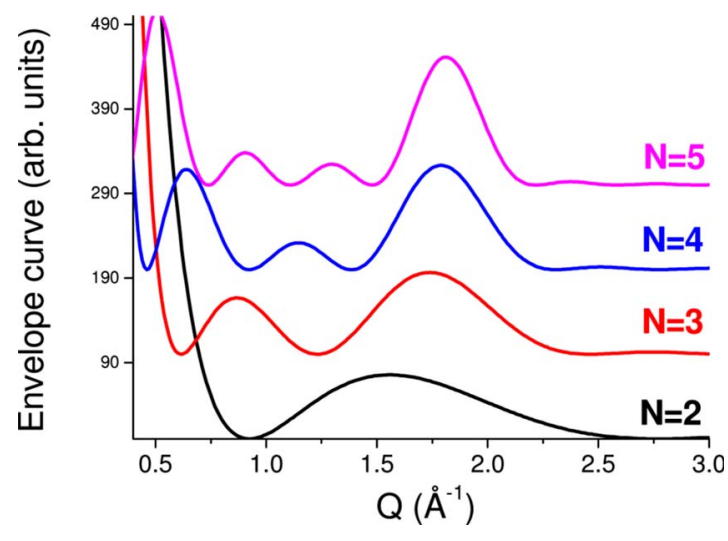

FIG. 5. (Color online) Calculated envelope curves (see text for details) of diffraction patterns of powders of isolated MWCNTs with $N=2,3,4$, and 5 walls; inner diameter is $13.2 \AA$ and intertube spacing is $3.4 \AA$.

analytically since, around $Q=\frac{2 \pi}{d R}$, for large $N$ values, one gets

$$
\left[\frac{\sin \left(\frac{N Q d R}{2}\right)}{\sin \left(\frac{Q d R}{2}\right)}\right]^{2} \approx N^{2} \exp \left(-\frac{N^{2}\left(\frac{Q d R}{2}-\pi\right)^{2}}{\pi}\right),
$$

which is a Gaussian function with FWHM $=\frac{2 \sqrt{\pi \ln 2}}{N}$, as plotted in Fig. 6.

\section{Bundled nanotubes}

Depending on the synthesis process, DWCNTs may be produced in isolated form or they may be found into bundles of variable size. In this part, we calculate the diffraction pattern of bundled DWCNTs as they can be found following the peapod conversion route and we determine the origin of the main features observed in this diffraction profile.

Calculations of the diffraction pattern of bundled DWCNTs powders can be achieved using the formalism shown in the previous paragraph, including a lattice term

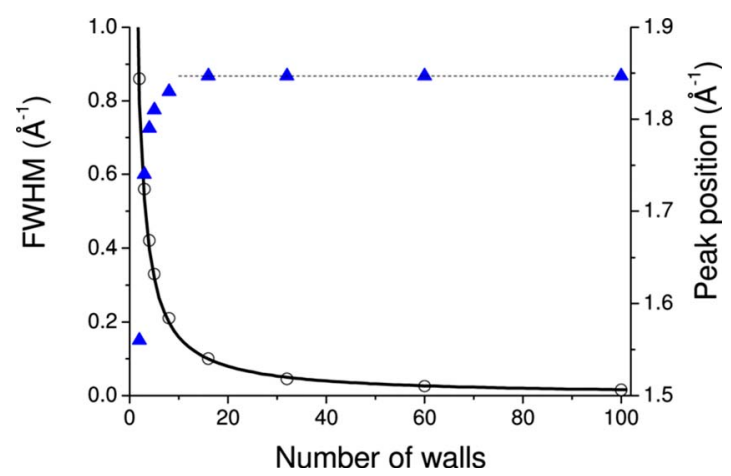

FIG. 6. (Color online) Open circles: full width at half maximum of the diffraction feature at $Q \sim 1.8 \AA^{-1}$ as a function of the number of walls of MWCNTs. Solid line: calculation of the width using the analytical expression coming from the development of the envelope curve into a Gaussian function $\left(\mathrm{FWHM}=\frac{2 \sqrt{\pi \ln 2}}{N}\right)$. Full triangles: position of the peak maximum; dashed line corresponds to $Q=\frac{2 \pi}{d R}$. 


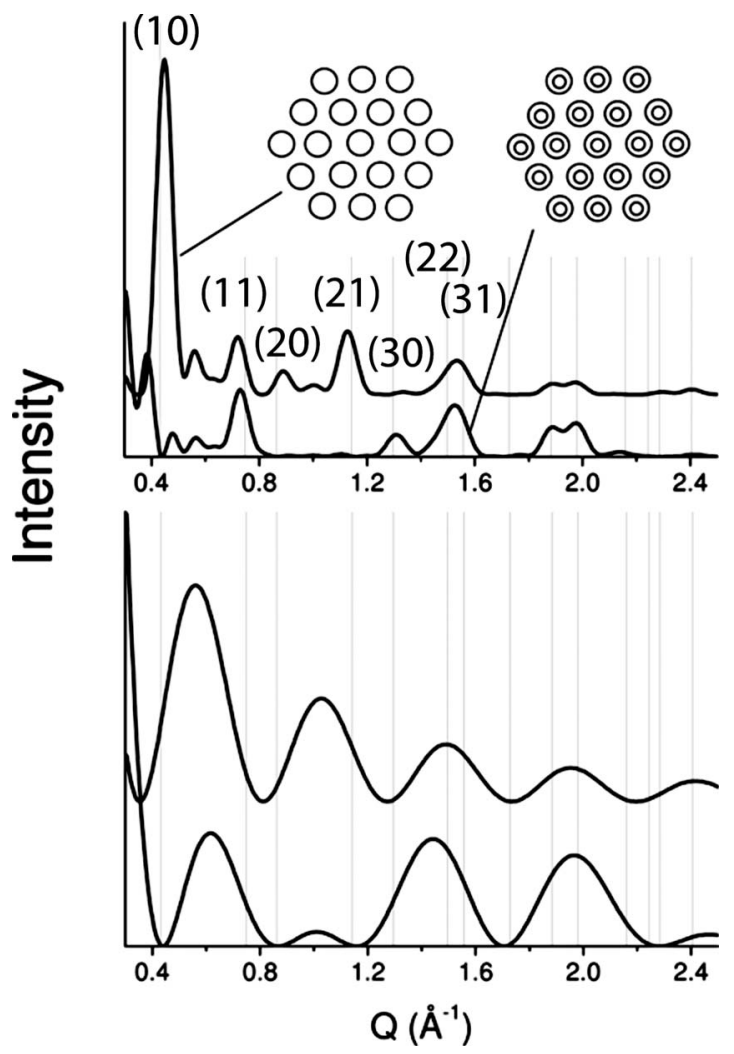

FIG. 7. Upper part: calculated diffraction patterns of a bundle of 19 SWCNTs of diameter $13.6 \AA$, with an intertube spacing $a_{\mathrm{vdW}}$ $=3.2 \AA$ and of a bundle of 19 DWCNTs, with outer diameter $D_{o}$ $=13.6 \AA$, an interwall distance $d R$ of $3.4 \AA$, and an inter-DWCNT spacing $a_{\mathrm{vdW}}$ of $3.2 \AA$. Lower part: calculations of the square of the term $T_{\mathrm{SW}}=D_{0} J_{0}\left(\frac{Q D_{0}}{2}\right)$ (top) and of the square of the term $T_{\mathrm{DW}}$ $=D_{i} J_{0}\left(\frac{Q D_{i}}{2}\right)+D_{0} J_{0}\left(\frac{Q D_{0}}{2}\right)$, respectively, involved in the calculation of the intensity scattered by a powder of SWCNTs and of DWCNTs. The vertical lines indicate the theoretical position of the Bragg peaks expected for the lattice of nanotubes in a bundle.

consisting in a sum over all the constitutive tubes of a bundle. The expression of the intensity diffracted by a powder of SWCNT bundles may be written as ${ }^{17-19}$

$$
I(Q) \propto \frac{\sigma^{2} f_{c}^{2}}{Q} \sum_{i, j}^{N_{t}} J_{0}\left(Q R_{i, j}\right)\left[D_{o} J_{0}\left(\frac{Q D_{o}}{2}\right)\right]^{2},
$$

where $N_{t}$ is the number of tubes forming the bundle and $R_{i, j}$ is the distance between tube $i$ and tube $j$ in the plane perpendicular to the bundle axis. The intensity diffracted by a powder of DWCNT bundles becomes

$$
I(Q) \propto \frac{\sigma^{2} f_{c}^{2}}{Q} \sum_{i, j}^{N_{t}} J_{0}\left(Q R_{i, j}\right)\left[D_{i} J_{0}\left(\frac{Q D_{i}}{2}\right)+D_{o} J_{0}\left(\frac{Q D_{o}}{2}\right)\right]^{2} .
$$

The upper part of Fig. 7 depicts the calculated diffraction profile of a powder of bundles made of 19 SWCNTs (i.e., two shells of nanotubes around a central one, giving an average bundle diameter of $70 \AA$ ), having a radius of $6.8 \AA$, and an intertube distance $a_{\mathrm{vdW}}$ of $3.2 \AA$, and that of a pow- der of bundles made of 19 DWCNTs, having an outer radius $R_{o}$ of $6.8 \AA$ and an intertube distance of $3.2 \AA$. Both DWCNT and SWCNT bundles have the same external dimensions, as represented on the picture in Fig. 7. Their diffraction patterns not only show similarities but also strong differences. The diffraction pattern of SWCNT bundles can be described as the association of the carbon nanotube form factor, mainly given by the oscillations of a $J_{0}$ Bessel function, and the Bragg reflections from the two-dimensional (2D) hexagonal lattice of the nanotubes in bundles. ${ }^{17,18}$ The most intense peak in the calculated diffraction pattern is the 10 reflection located at $\sim 0.4 \AA^{-1}$ and at higher $Q$ values the $11,20,21$, and $22+31$ reflection can be observed at 0.75 , $0.9,1.1$, and $1.5 \AA^{-1}$, respectively. In the case of DWCNT bundles, the 11 and $22+31$ peaks are still clearly observable, at about the same position than for SWCNTs. This is expected since the crystalline lattice and the lattice parameter of DWCNTs are the same than in the case of SWCNTs. However, some reflections have disappeared, the most spectacular extinction being that on the (10) planes.

It was demonstrated in previous studies that the filling of bundled nanotubes with diameter around $14 \AA$ by a variety of materials such as gas, ${ }^{24}$ inorganic compounds, ${ }^{25,26}$ and carbonaceous materials such as $\mathrm{C}_{60}$ in peapods ${ }^{19}$ can dramatically affect the intensity and the position of the 10 reflection. The same phenomenon occurs for DWCNTs. It is due to the difference between the terms $T_{\mathrm{SW}}^{2}$ and $T_{\mathrm{DW}}^{2}$, respectively, associated with single- and double-walled nanotubes, as shown in Fig. 7. Note that the changes in these terms versus $Q$ also imply, for the considered tubes of external diameter around $14 \AA$, a strong diminution of the 21 reflection intensity.

The conversion of peapods into DWCNTs always results in "incomplete" DWCNTs with shorter inner tubes compared to outer ones. As a consequence and similarly to the case of $\mathrm{C}_{60}$ peapods, calculations of XRD patterns of DWCNTs with shorter inner tubes would result in the reappearance of the 10 and 21 peaks. The scattered intensity may be written as

$$
I(Q) \propto \frac{\sigma^{2} f_{c}^{2}}{Q} \sum_{i, j}^{N_{t}} J_{0}\left(Q R_{i, j}\right)\left[\alpha D_{i} J_{0}\left(\frac{Q D_{i}}{2}\right)+D_{o} J_{0}\left(\frac{Q D_{o}}{2}\right)\right]^{2},
$$

where $\alpha$ is the ratio between the lengths of the internal and external tubes (for conversion from $\mathrm{C}_{60}$ peapods, with filling rate $p$ and interfullerene distance $L=9.8 \AA$, one finds, assuming conservation of the number of atoms inside the outer tube, $\left.\alpha=\frac{60 p}{\pi \sigma D_{i} L}\right)$. It is important to take this factor into account because the conversion of peapods into DWCNTs always results in this kind of incomplete DWCNTs.

Our calculations therefore demonstrate that the XRD pattern of bundled DWCNTs show "accidental" extinctions of some Bragg peaks characteristic of the 2D hexagonal lattice of bundles, which must be carefully analyzed by simulating the XRD patterns in order to derive relevant structural characterization of bundled DWCNT samples. 


\section{B. Experiments}

In this part, we use the analytical expressions detailed above to obtain a description of real DWCNT samples, synthesized by CCVD or peapod conversion.

\section{Sample synthesis}

CCVD-produced samples ${ }^{7}$ were synthesized using $\mathrm{MgO}-$ based catalysts prepared by combustion synthesis. The catalyst was reduced in a $\mathrm{H}_{2}-\mathrm{CH}_{4}$ mixture $\left(18 \mathrm{~mol} \% \mathrm{CH}_{4}\right.$, heating and cooling rates of $5{ }^{\circ} \mathrm{C} / \mathrm{min}$, maximum temperature of $1000{ }^{\circ} \mathrm{C}$, and no dwell), resulting in a dense mat of DWCNT composite powder. Catalyst dissolution was achieved by addition of $\mathrm{HCl}$, followed by extensive washing with deionized water. TEM observations and infrared-absorption spectroscopy analyses ${ }^{7,27}$ have shown that no amorphous carbon could be found in significant quantity in this sample. Checking the absence of amorphous carbon by-products by different methods prior to the XRD analysis of DWCNT samples is of major importance since amorphous carbon gives a large peak around $1.6 \AA^{-1}$. Such a peak would seriously complicate the determination of the number of walls of the tubes in the sample.

The starting materials for DWCNTs prepared from the peapods conversion route are arc-discharge SWCNT powders from Nanocarblab, Incorporated. The powder was subjected to a purification procedure which included an oxidizing thermal treatment, a nitric acid treatment, and a $800{ }^{\circ} \mathrm{C}$ annealing in inert atmosphere. The powder contains $\sim 30 \%$ of nontubular carbon nanophases, which have withstood the purification procedure. The latter has resulted in the opening of the nanotubes. The final annealing temperature $\left(800{ }^{\circ} \mathrm{C}\right)$ is high enough to eliminate adsorbed organic impurities and sufficiently low to prevent the closure of the nanotubes. First we prepared $\mathrm{C}_{60}$ peapods following the vapor filling method whose principle was initially proposed in Ref. 3. Basically, we sublimed $\mathrm{C}_{60}$ powder in excess at $450{ }^{\circ} \mathrm{C}$ in the presence of the opened SWCNTs in sealed quartz tubes, and maintained it for $24 \mathrm{~h}$. After the synthesis, peapods were heated up at $1300{ }^{\circ} \mathrm{C}$ during $48 \mathrm{~h}$ in dynamic vacuum to transform $\mathrm{C}_{60}$ peapods into DWCNTs. The remaining (i.e., free) $\mathrm{C}_{60}$ molecules which had not entered the SWCNTs during the filling process were removed during the heating ramp up to $1300{ }^{\circ} \mathrm{C}$.

XRD experiments were performed using the $K \alpha$ emission of a $\mathrm{Cu}$ rotating anode $(\lambda=1.5418 \AA)$ at room temperature and recorded on an image plate. The signal/background ratio was improved using a sample holder made of a $10-\mu$ m-thick aluminum foil instead of a glass capillary, whose amorphous signal around $1.5 \AA^{-1}$ would be very disturbing when studying the DWCNT signal. This aluminum sample holder does not give additional signal in the $Q$ range of the study from 0.3 to $2.5 \AA^{-1}$. Moreover experiments are done under vacuum to eliminate the diffuse scattering of air.

\section{Comparison between experimental and theoretical patterns}

Figure 8 shows a comparison between an experimental XRD pattern of CCVD-produced DWCNTs and the result of our diffraction pattern calculation. Note that the calculations

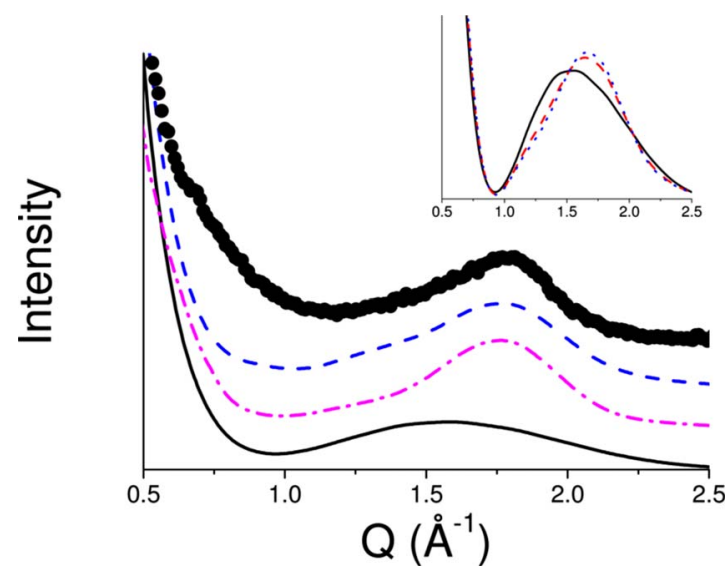

FIG. 8. (Color online) Plain circles: XRD pattern of a sample of CCVD-produced DWCNTs. Plain line: calculation involving a mixture of SWCNTs, DWCNTs, and MWCNTs with proportions estimated from TEM (see text for details). Dashed line: calculation featuring isolated DWCNTs and MWCNTs giving the best agreement with the experimental profile (see text for detail). Dasheddotted line: calculation featuring bundles of 19 DWCNTs and isolated MWCNTs giving the best agreement with the experimental profile (see text for details). Inset: calculation of the XRD pattern of pure DWCNT with a mean radius of $7 \AA$, a radii distribution of FWHM $=6 \AA$, and $d R=3.4 \AA$ with isolated DWCNTs (plain line), bundles of 7 DWCNTs (dashed line), and bundles of 19 DWCNTs (dotted line).

were performed in agreement with the formula we presented above and that two experimental factors that should be taken into account for a direct comparison with experimental data have been added to the formula: (i) a geometrical factor $G(\theta)=\cos ^{3}(2 \theta)$ taking into account the detection on the planar geometry of an imaging plate and (ii) the polarization factor $P(\theta)=\frac{1+f_{m}(\theta) \cos ^{2}(2 \theta)}{2}$, where $f_{m}(\theta) \sim 1$ and depends on the monochromator.

The comparison between our simulations and the experimental diffraction profiles is made on the basis of profile matching, without the use of the reliability factor ( $R$ factor) commonly used in powder-diffraction pattern fitting. The reason for that is that the diffraction features to be considered are very low in intensity with regard to the background; moreover, they are rather large. The $R$ factor would therefore become overdependent on the background correction, considerably limiting its relevance. In fact, Rietveld analysis of a SWCNT sample has only been published by one team, ${ }^{28}$ for samples formed of large bundles of SWCNT with a narrow diameter distribution, corresponding to an optimal case. Even in this case, it did not give more information than qualitative comparison of profile matching. We have attempted to perform reliability factor minimization and we reached the same conclusion.

In order to perform the calculations, we started from the diameter distributions estimated by a semiquantitative study performed by TEM observations on $\sim 100$ tubes. $^{7}$ The mean diameter of the inner tubes was found to be of $\sim 13 \AA$, with a very large diameter distribution showing a FWHM of $12 \AA .{ }^{29}$ The intertube spacing is estimated to be $3.4 \AA$. A tentative composition of the sample was also deduced from 
TEM experiments, featuring $18 \%$ of SWCNTs, $77 \%$ of DWCNTs, and $5 \%$ of triple-walled carbon nanotubes (3WCNTs). Figure 8 shows the calculated diffraction pattern of a sample featuring such a composition (bottom plain line). A comparison with the experimental pattern shows that the calculated profile presents a large feature around $1.6 \AA^{-1}$, in poor agreement with the experimental data. The excessive width of this first attempt seems due to an overestimation of the proportion of DWCNTs in the sample since the shape of DWCNTs is similar to that of the fitting curve. A more satisfying profile for the fitting curve would imply a stronger proportion of tubes with a larger number of walls, 3WCNTs, tetrawalled carbon nanotubes (4WCNTs), and pentawalled carbon nanotubes (5WCNTs), in order to sharpen the peak and to shift it to its experimental value around $1.8 \AA^{-1}$. It has to be noted that the proportion of SWCNTs is very hard to determine from experimental data. The diffraction signal from SWCNTs is indeed mostly concentrated below $Q$ $=1 \AA^{-1}$, where signals mainly coming from sample porosity superimpose with that of SWCNTs, rendering the estimation of SWCNT amount very imprecise. In consequence, we focused on the fit of the large feature around $1.8 \AA^{-1}$ to estimate the proportion of MWCNT populations. The dashed curve in Fig. 8 shows a fit which gives a better agreement with the experimental profile. It involves DWCNTs of same dimension than that considered in previous calculations, but the proportions of MWCNT populations are modified as follows: 70\% DWCNTs, 20\% 3WCNTs, 5\% 4WCNTs, and 5\% 5WCNTs. The dashed-dotted line in Fig. 8 displays the result of another calculation featuring bundled DWCNTs. The reason for this hypothesis is that bundled DWCNTs were sometimes observed using TEM. ${ }^{7}$ The inset in Fig. 8 shows that as soon as bundled DWCNTs are considered, and that the DWCNT diameter distribution remains large, the width of the feature at $1.8 \AA^{-1}$ is slightly decreased and its position is shifted to higher $Q$ values. ${ }^{30}$ In consequence, the amount of DWCNTs could be maximized when fitting the feature at $1.8 \AA^{-1}$ with bundled DWCNTs. In our calculations, a good agreement was found at the cost of a slight modification of MWCNT proportions: $80 \%$ DWCNTs, $10 \%$ 3WCNTs, 5\% $4 \mathrm{WCNTs}$, and 5\% 5WCNTs. Taking into account this small variation depending of the aggregation state of the nanotubes, and the changes observed in our different attempts to fit the data, we estimate the error in our determination of the MWCNT proportions to be of about $10 \%$. The simulation of XRD profiles therefore allowed a valuable description of the sample at the macroscopic scale to be given and appears fully complementary to local-scale investigations such as those using TEM or other local probes.

Figure 9 shows the experimental diffraction diagram of a DWCNT sample from the peapods conversion route. This diffraction pattern is dramatically different from that of samples synthesized by CCVD methods. The observation of peaks located at $0.4,0.75$, and $1.45 \AA^{-1}$ strongly suggests that the DWCNTs are stacked into bundles (see Fig. 7) and present a quite narrow diameter distribution. We present in Fig. 9 the result of a calculation in good agreement with the experimental diffraction profile, involving outer tubes of mean radius $7.1 \AA$, with a distribution of $1 \AA$ FWHM. All

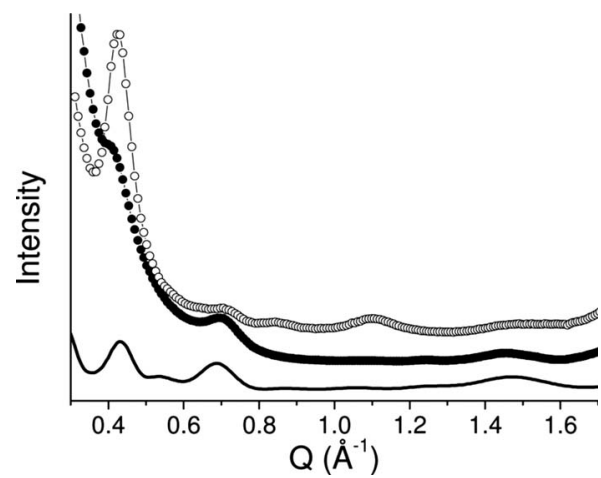

FIG. 9. Open circles: experimental diffraction pattern of the reference SWCNT sample for peapod synthesis. Filled circles: experimental diffraction pattern of the DWCNT sample from peapod conversion. Plain line: calculation of the diffraction pattern of DWCNTs giving a good fit with the experimental data (see text for details).

tubes are packed into 19-tube bundles, which correspond to an average bundle diameter of $70 \AA$, and they are separated by a distance of $3.2 \AA$. These parameters are derived from the diffraction pattern of nanotubes precursors. As in Ref. 15, the inner-tube radius in the peapod-derived DWCNTs is chosen equal to that of the external tube minus $3.6 \AA$, which is the van der Waals distance at the high temperature corresponding to the inner-tube formation. The calculation is made on the basis of an initial peapod sample filled at $80 \%$ with $\mathrm{C}_{60}$ molecules. According to the diameter of the inner tube and to the filling rate at the sample scale, the average ratio $\alpha$ of inner-tube length over outer-tube length in final DWCNTs is $\sim 0.6$. The good agreement between the experimental data and the calculations illustrates the validity of the model presented here to describe also DWCNT samples formed of large bundles of nanotubes with well-defined diameters. It is in agreement with the results of the authors of Refs. 15 and 16 who used an approximation of the model valid in this case.

The XRD investigation of these two DWCNT samples originating from different synthesis routes points out large differences in their structures. CCVD-produced DWCNTs that grow directly on catalytic substrates present a rather disordered structure and contain other types of tubes than DWCNTs. They contain tubes presenting a large diameter distribution. On the contrary, DWCNTs from peapod conversion are well ordered in large bundles, with a narrow diameter distribution, but the incomplete filling of nanotubes by $\mathrm{C}_{60}$ leads to internal tubes shorter than the external ones.

\section{CONCLUSION}

In the first part of this study, we detailed the formalism allowing the calculation of the powder-diffraction pattern of DWCNTs. We gave an analytical description of the main features appearing in the diffraction pattern of isolated DWCNTs and we further studied the way these features evolve with the main structural parameters of DWCNTs (tubes radii and intertube spacing). We then considered the 
diffraction pattern of bundles of DWCNTs and we gave an explanation for the accidental extinction of some of the Bragg peaks relative to the structure of the tubes forming bundles.

In the second part of this study, we compared experimental diffraction patterns of DWCNT samples originating from two different routes: CCVD and peapod conversion. We managed to fit the experimental profile in both cases, exem- plifying the general use that can be made of the structural model.

Through these detailed calculations and these examples, we gave to experimentalists concerned with the characterization of macroscopic amounts of DWCNT samples all the tools necessary to take advantage of XRD for a global determination of the composition and structure of their nanotube samples.
*Corresponding author. FAX: +331 691560 86;

cambedouzou@1ps.u-psud.fr

${ }^{1}$ G. Chen, S. Bandow, E. R. Margine, C. Nisoli, A. N. Kolmogorov, V. H. Crespi, R. Gupta, G. U. Sumanasekera, S. Iijima, and P. C. Eklund, Phys. Rev. Lett. 90, 257403 (2003).

${ }^{2}$ J. Cambedouzou, J.-L. Sauvajol, A. Rahmani, E. Flahaut, A. Peigney, and C. Laurent, Phys. Rev. B 69, 235422 (2004).

${ }^{3}$ B. W. Smith and D. E. Luzzi, Chem. Phys. Lett. 321, 169 (2000).

${ }^{4}$ A. G. Souza Filho, V. Meunier, M. Terrones, B. G. Sumpter, E. B. Barros, F. Villalpando-Páez, J. Mendes Filho, Y. A. Kim, H. Muramatsu, T. Hayashi, M. Endo, and M. S. Dresselhaus, Nano Lett. 7, 2383 (2007).

${ }^{5}$ R. Saito, R. Matsuo, T. Kimura, G. Dresselhaus, and M. S. Dresselhaus, Chem. Phys. Lett. 348, 187 (2001).

${ }^{6}$ A. Hashimoto, K. Suenaga, K. Urita, T. Shimada, T. Sugai, S. Bandow, H. Shinohara, and S. Iijima, Phys. Rev. Lett. 94, 045504 (2005).

${ }^{7}$ E. Flahaut, R. Bacsa, A. Peigney, and C. Laurent, Chem. Commun. (Cambridge) 2003, 1442.

${ }^{8}$ M. Endo, H. Muramatsu, T. Hayashi, Y. A. Kim, M. Terrones, and M. S. Dresselhaus, Nature (London) 433, 476 (2005).

${ }^{9}$ J. L. Hutchison, N. A. Kiselev, E. P. Krinichnaya, A. V. Krestinin, R. O. Loutfy, A. P. Morawsky, V. E. Muradyan, E. D. Obraztsova, J. Sloan, S. V. Terekhov, and D. N. Zakharov, Carbon 39, 761 (2001).

${ }^{10}$ S. Bandow, M. Takizawa, K. Hirahara, M. Yudasaka, and S. Iijima, Chem. Phys. Lett. 337, 48 (2001).

${ }^{11}$ B. W. Smith, M. Monthioux, and D. E. Luzzi, Nature (London) 396, 323 (1998).

${ }^{12}$ M. Monthioux, E. Flahaut, and J.-P. Cleuziou, J. Mater. Res. 21, 2774 (2006)

${ }^{13}$ J.-F. Colomer, L. Henrard, P. Launois, G. Van Tendeloo, A. A. Lucas, and Ph. Lambin, Phys. Rev. B 70, 075408 (2004).

${ }^{14}$ R. Pfeiffer, F. Simon, H. Kuzmany, and V. N. Popov, Phys. Rev. B 72, 161404(R) (2005).

${ }^{15}$ M. Abe, H. Kataura, H. Kira, T. Kodama, S. Suzuki, Y. Achiba,
K. I. Kato, M. Takata, A. Fujiwara, K. Matsuda, and Y. Maniwa, Phys. Rev. B 68, 041405(R) (2003).

${ }^{16}$ R. Pfeiffer, M. Holzweber, H. Peterlik, H. Kuzmany, Z. Liu, K. Suenaga, and H. Kataura, Nano Lett. 7, 2428 (2007).

${ }^{17}$ A. Thess, R. Lee, P. Nikolaev, H. Dai, P. Petit, J. Robert, C. Xu, Y. H. Lee, S. G. Kim, A. G. Rinzler, D. T. Colbert, G. E. Scuseria, D. Tománek, J. E. Fischer, and R. E. Smalley, Science 273, 483 (1996).

${ }^{18}$ S. Rols, R. Almairac, L. Henrard, E. Anglaret, and J.-L. Sauvajol, Eur. Phys. J. B 10, 263 (1999).

${ }^{19}$ J. Cambedouzou, V. Pichot, S. Rols, P. Launois, P. Petit, R. Klement, H. Kataura, and R. Almairac, Eur. Phys. J. B 42, 31 (2004).

${ }^{20}$ M. Chorro, A. Delhey, L. Noé, M. Monthioux, and P. Launois, Phys. Rev. B 75, 035416 (2007).

${ }^{21}$ J.-F. Colomer, L. Henrard, E. Flahaut, G. Van Tendeloo, A. A. Lucas, and Ph. Lambin, Nano Lett. 3, 685 (2003).

${ }^{22}$ M. Kociak, K. Hirahara, K. Suenaga, and S. Iijima, Eur. Phys. J. B 32, 457 (2003).

${ }^{23}$ B. E. Warren, X-Ray Diffraction (Dover Publications, New York, 1990).

${ }^{24}$ S. Rols, M. R. Johnson, P. Zeppenfeld, M. Bienfait, O. E. Vilches, and J. Schneble, Phys. Rev. B 71, 155411 (2005).

${ }^{25}$ N. Bendiab, R. Almairac, S. Rols, R. Aznar, J.-L. Sauvajol, and I. Mirebeau, Phys. Rev. B 69, 195415 (2004).

${ }^{26}$ J. Cambedouzou, S. Rols, N. Bendiab, R. Almairac, J.-L. Sauvajol, P. Petit, C. Mathis, I. Mirebeau, and M. Johnson, Phys. Rev. B 72, 041404(R) (2005).

${ }^{27}$ J.-L. Bantignies, J.-L. Sauvajol, A. Rahmani, and E. Flahaut, Phys. Rev. B 74, 195425 (2006).

${ }^{28}$ H. Kadowaki, A. Nishiyama, K. Matsuda, Y. Maniwa, S. Suzuki, Y. Achiba, and H. Kataura, J. Phys. Soc. Jpn. 74, 2990 (2005).

${ }^{29}$ In order not to consider inner tubes with a too small diameter, we did not take into account tubes of diameter smaller than $3 \AA$.

${ }^{30}$ Calculated diffraction patterns of bundled MWCNTs with more than two walls did not show any significant differences from that of isolated MWCNTs. 